affray. Diagnoses included learning disability, delusional disorder, paranoid schizophrenia, bipolar affective disorder, alcohol dependence, personality disorder and depressive disorder.

Patients had multiple comorbidities such as diabetes, COPD, hypertension, coronary artery disease and musculoskeletal problems. Out of the nine admitted patients, only six had an ACE with an average score of 70.83 . Five patients had brain imaging, with two normal results and the others showing some degree of atrophy and ischemic changes.

Discharge destinations included medium secure units, low secure unit and prison. One patient unfortunately died during admission and four are still inpatients.

A staff survey conducted showed their perspective on the challenges in managing elderly patients and whether Wathwood Hospital had the appropriate resources for them to work with elderly offenders in their area of work. All results will be explained through tables and graphs.

Conclusion. It's evident that there are challenges in managing elderly patients in units not specifically designed to manage them. This is also due to the lack of geriatric training and resources available to allied health care professionals to carry out their respective work. It's therefore crucial we formulate more inclusive strategies to address these challenges.

The use of antipsychotic polypharmacy at Ravenswood House Medium Secure Unit: the extent of use and reporting of outcomes

Laura Cherrington* and Hari Patel

Ravenswood House Medium Secure Unit

${ }^{\star}$ Corresponding author.

doi: 10.1192/bjo.2021.828

Aims. To evaluate the use of antipsychotic polypharmacy in Ravenswood House Medium Secure Hospital. We also aimed to review the reporting of the outcomes of their use.

Background. The use of antipsychotic polypharmacy (APP) continues to be practised within forensic psychiatric inpatient settings yet there is a lack of robust evidence for the benefits of doing so. The practice is also associated with the use of higher total antipsychotic doses beyond the recommended BNF maximum. Such prescribing is associated with an increased side effect burden. Doctors have a duty to justify the ongoing use of antipsychotic polypharmacy and to avoid potentially ineffective and/or harmful use.

Method. A cross-sectional review of the medication cards for 51 in-patients at Ravenswood House Hospital was completed. Demographic data and data pertaining to diagnoses and medication was also gathered from the electronic patient records.

Result. 23 patients (45\%) in Ravenswood House Hospital were prescribed antipsychotic polypharmacy. $87 \%$ of those prescribed antipsychotic polypharmacy had a primary diagnosis of either schizophrenia or schizoaffective disorder. 19 patients (37\%) had two regular antipsychotics prescribed. $74 \%$ of these prescriptions were above the recommended BNF maximum. $62 \%$ were also prescribed a regular benzodiazepine. The vast majority of indications documented for APP were chronic behavioural disturbance and treatment resistant schizophrenia. The majority of these patients were on a T3. There was a significant under reporting of the rationale of prescribing APP. It could be surmised that at least 11 combinations were in part to mitigate side effects, but only 3 had this documented. There was also a lack of documentation or use of rating scales regarding the clinical outcomes and side effects of APP.

Conclusion. Prescription of antipsychotic polypharmacy is an important issue in secure forensic hospital settings. The lack of clear documentation of clinical effectiveness and side effect burden remains a concern. Wider study is required to establish the benefits of such prescribing to justify its ongoing use.

\section{Capacity and consent to treatment - how well did we do?}

Khui Chiang Wee*, Nithya Anandan, Nguemo Angahar and Abhilash Mannam

Northwest Boroughs HealthCare NHSFT

${ }^{\star}$ Corresponding author.

doi: 10.1192/bjo.2021.829

Aims. An audit on capacity assessment and consent to treatment on inpatient visits to Atherleigh Park Hospital was performed using the Mental Health Act Code of Practice as a framework. Six standards were evaluated:

1) documentation of capacity assessment in patient care records

2) documentation of patients who display a lack of capacity

3) completion of a Section 58 and/or 62 for detained patients

4) documentation of medicines on T2/T3 form and if they match with the patient's prescription chart

5) evidence of medication concordance and monitoring of adverse side effects

6) patient education on medicines prescribed for them

Method. Inclusion criteria included patients who were detained under Sections 2, 3 and informal admissions, who were admitted for 72 hours or more, between October and December 2019. This gave a total sample size of 75 . Data were collected by looking at patients' care records and if applicable, their Section paperwork to identify any documentations related to the standards evaluated as above. Data collected were transcribed to a web link, downloaded and analysed.

Result. In standard 1), it was found that $77 \%$ of the capacity assessment and consent to treatment forms were recorded in patient care records. Of these, $100 \%$ of were completed by a medic and $99 \%$ of all sections in the form were completed. However, only $57 \%$ of patients were re-assessed when their capacity and consent changed during admission. In standards 2), 3 ) and 4), documentation of patients who lacked capacity, completion of a Section 58 or 62 form and charting of medications on the T2/T3 forms were fully compliant. In standards 5) and 6), $76 \%$ of medication concordance were documented in patients' records. Only $39 \%$ of adverse effects from medications were documented but monitoring compliance was $100 \%$. Medication counselling was done infrequently, with $47 \%$ of patients given a leaflet and $28 \%$ educated on their side effects.

Conclusion. Action plans were identified. Firstly, to link the capacity assessment form with patient electronic ward round notes to ensure clinicians complete it at the end of a review. In order to monitor adverse effects from medications, physical examination, blood tests and ECG are to be done following a new prescription, and to be repeated if indicated. Information leaflets on common psychiatric medications are to be made readily available for patients. The findings from this service evaluation and the actions plans were shared with doctors. A re-audit is vital to re-evaluate the hospital's compliance. 